\title{
Visual Verification Method for Indirect Line of Sight Stabilization
}

\author{
Zhu-Lin Wang, Wei-Wei Gao, Peng He, Hong-Yun Wang \\ Four Department, Ordnance Engineering College, Shijiazhuang, China \\ E-mail: swcwzl@126.com, weiweizhiwa@126.com
}

\begin{abstract}
In order to visualize the validation results of Indirect Line of sight stabilization, Visual simulation research is carried out. In the $\mathrm{VC}++$ environment, the visual simulation platform is built by combining the OpenGL and MFC technology. The target and field of view are simulated. Process of Indirect Line of sight stabilization before and after error correction are visualized. The results show that the Visual simulation platform can be used to realize the verification for the correlate algorithm and model as Indirect Line of sight stabilization.
\end{abstract}

Keywords- indirect line of sight stabilization; visual simulation;openGL; MFC.

\section{INTRODUCTION}

Visual simulation technology has become a hot spot in the field of simulation in recent years due to the visual presentation of simulation results. In the research field of Line of sight stabilization(LOS), the research is less on the combination of LOS and visual simulation technology. In the research of the related model and algorithm to indirect LOS, to verify the theoretical results, and make theoretical research results more intuitive, this paper carried out the research of visual simulation for indirect LOS. Aiming to build stable visual simulation platform, the relevant algorithm and model embedded, makes visualization result of the algorithm.

\section{VISUAL SIMULATION TECHNOLOGY RELATED TO INDIRECT LOS}

Based on the research of the target airspace LOS as an example, we first need to establish 3D models such as spatial object model (this paper represented by the plane model), sighting equipment field model. Secondly, it is need to establish a software environment, and realize reading, display and control of the models in the software environment. Finally, it is need to be combined with the actual data and simulation environment, and realize the simulation for the state and environment of model in air defense test conditions, and eventually realize the verification and evaluation of system performance[1-3].

In the aspect of $3 \mathrm{D}$ visual simulation, comprehensive advantages of 3dsmax and OpenGL, we first established model of the system simulation platform by $3 \mathrm{dsmax}$, then the model is transformed into a OpenGL program which can be used to implement control[4-7]. Because OpenGL is 3D the underlying graphics graphics library, can not be directly used to describe the scene, and the Windows application framework can be used under the environment of $\mathrm{VC}++$
MFC, the display and control of 3D model can be achieved using rich window and event management function to call the OpenGL function [8-10].

In conclusion, the visual simulation platform scheme for indirect LOS is shown in Figure 1. Using 3dsmax software to establish the 3D model of .3ds format, and converted to.H and.Gl which is readable by View3ds. To establish a framework based on the MFC simulation platform in the $\mathrm{VC}++$ environment, render and initialize OpenGL environment under the framework. By loading the model and reading the OpenGL program of the model information, or by loading the measured data, to achieve control of the model such as speed, position and attitude. Ultimately implement the visual simulation, and the system performance evaluation for indirect LOS.

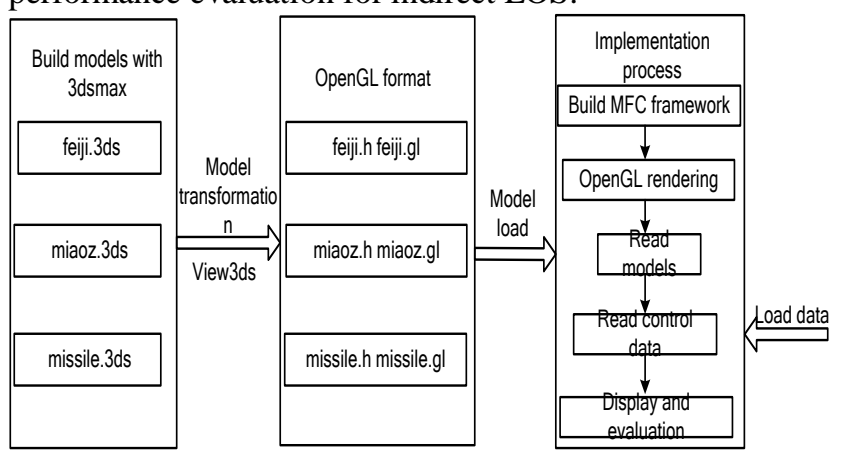

Figure 1. The realization scheme of the visual simulation platform of stabilized aiming system.

The above scheme is used to obtain the LOS visual simulation platform interface as shown in figure 2.

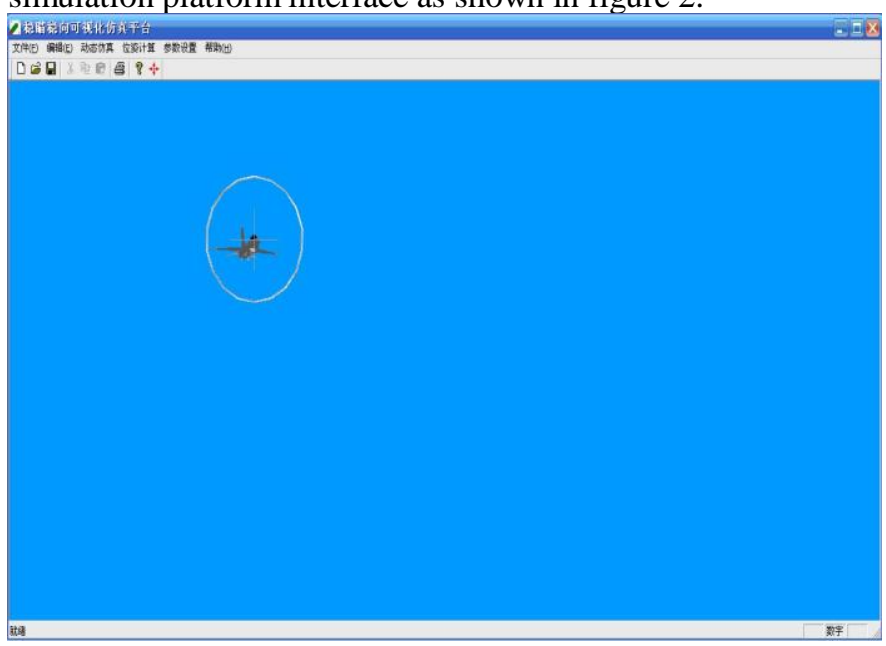

Figure 2. The visual simulation platform interface. 


\section{VISUAL SIMULATION VERIFICATION SCHEME FOR INDIRECT LOS}

With a certain type of equipment of photoelectric equipment target aiming process to the flying as an example, in a given target flight trajectory, to attack targets accurately, photoelectric aiming field should always track moving targets, until the missile hit the target. In the actual defense operations, sighting equipment is mounted on the moving carrier, and moving carrier attitude maneuver will affect tracking and aiming process, after adding stabilizing device, carrier attitude calculation error and axial error will cause the attitude error compensation for indirect LOS, which will lead to aimed at the field of view is not stable in the target, and visualization platform embodied at ring cross center deviated from the target. In the case of the error correction method of the strapdown attitude measurement under the condition of the moving carrier, the specific verification scheme is shown in Figure 3.

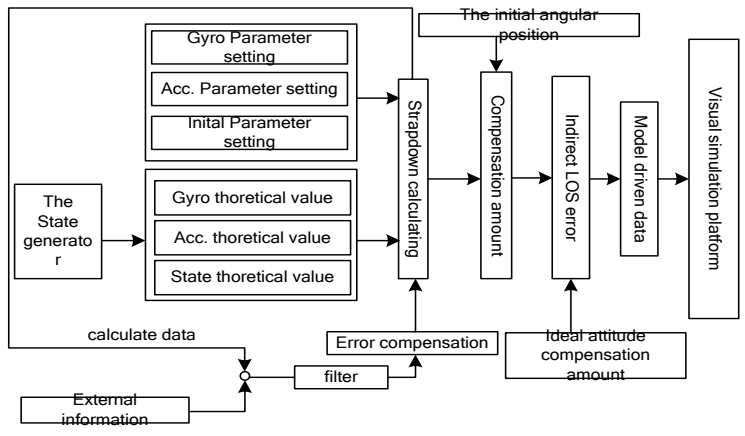

Figure 3. The visual verification scheme of indirect LOS.

Firstly, through the moving vehicle maneuvering state generator, inertial and moving vehicle state parameters of theoretical value is generated. After setting the relevant parameters error, stable attitude compensation is calculated, and the calculated attitude under error or without error input conditions were compared, so that steady error is obtained. The error data drives photoelectric aiming ring model, and finally intuitive steady effect is shown through the visualization simulation platform. By using the optimal estimation method to estimate the errors which external sources of information assisted, and get the corrected steady error, the error is introduced to show in the visual simulation platform, by comparing the display results with error and after error correction, to verify the effect of error correction.

\section{VISUAL SIMULATION VERIFICATION FOR INDIRECT LOS}

The distance between flight target and sighting equipment on the moving carrier is $2000 \mathrm{~m}$, actual flight target size can be regarded as a sphere of radius $5 \mathrm{~m}$, to capture the target aiming in field, attitude compensation error can not be more than $0.2865 \mathrm{deg}$. In the targeting process, pointing error changes caused by the position of the moving carrier compensate by controlling photoelectric aiming axial position. The main aiming is to isolate moving carrier attitude maneuver effect of targeting process. Assume that the initial reference system is set coincides with the reference system, Capturing target equipment, azimuth angle of the axial in the load system is 0 degrees, the pitch angle is 60 degrees. In the targeting process, the axial orientation in the frame of reference angle ranges from $0 \mathrm{deg} \sim 45 \mathrm{deg}$, pitch range is $60 \sim 30$ degrees. The zero offset of gyro is 1 degrees $/ \mathrm{h}$, and the zero offset of accelerometer is $1000 \mu \mathrm{g}$, the initial attitude error is $30^{\prime}$, the initial velocity error is $1 \mathrm{~m} / \mathrm{s}$. In the process of the attitude maneuver of the moving carrier, the tracking process of the photoelectric aiming equipment to the target is shown in Figure 4 .Under the condition of no error input, the tracking process of photoelectric aiming equipment is as shown in Figure 5. Using velocity and attitude as the external reference information source, the error parameters of the strapdown attitude measurement are corrected. After the correction, the visual simulation result is shown in Figure 6.

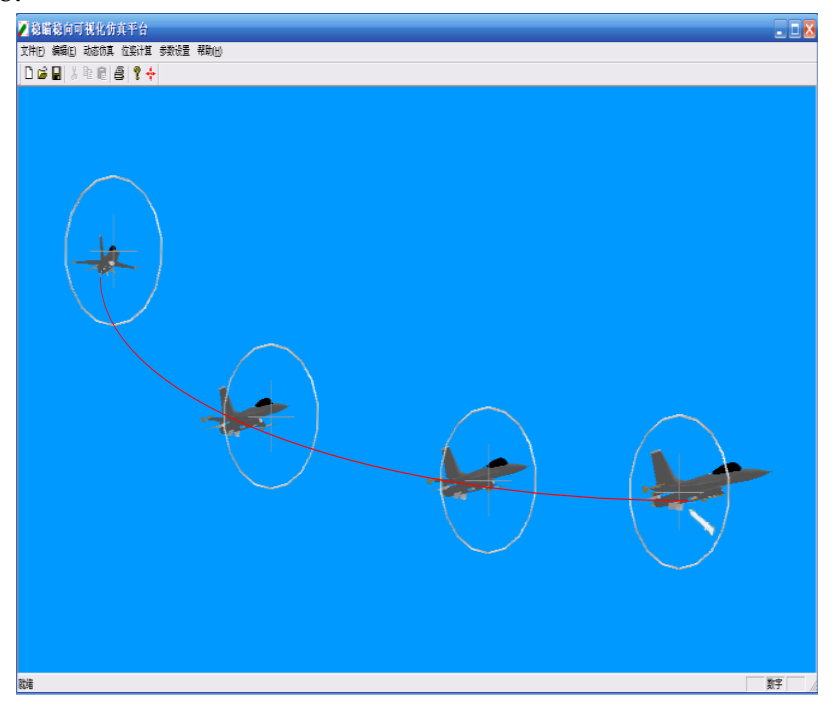

Figure 4. The tracking process of the target tracking device with no error.

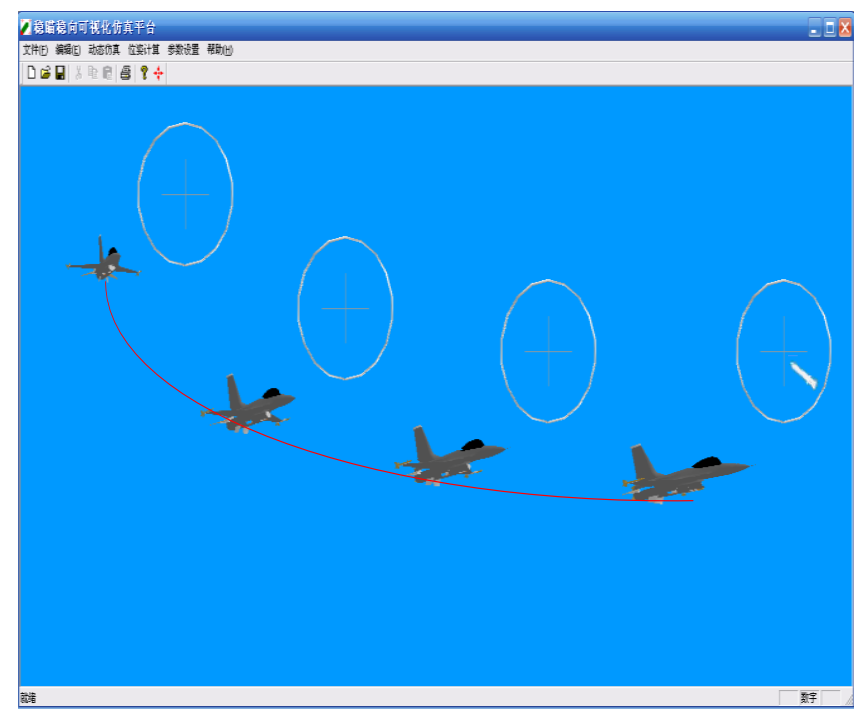

Figure 5. The tracking process of the target tracking device with the input error. 


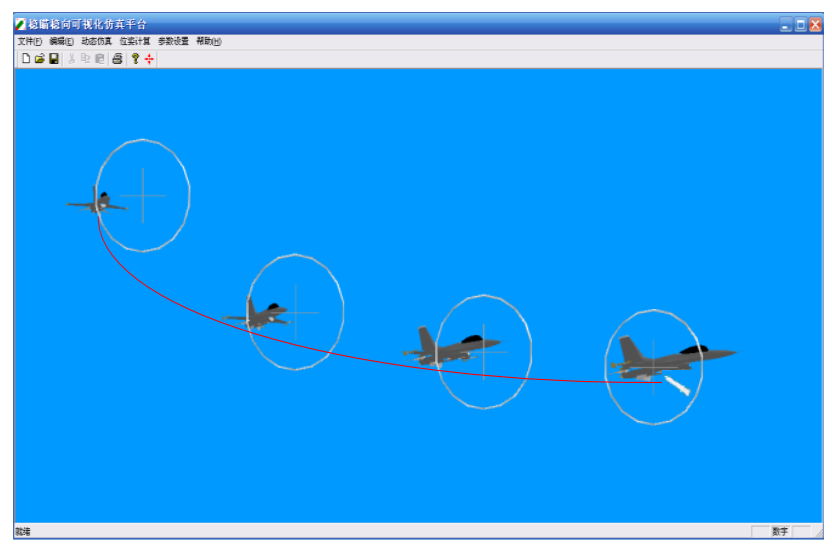

Figure 6. The tracking process of photoelectric aiming equipment after error correction

By comparing the tracking process before and after error correction, the effect of error correction algorithm can be evaluated directly. Visual simulation platform of indirect LOS can make result of the theory and method more intuitive. On the basis of perfecting relevant model and theory algorithm, the visual simulation platform of indirect LOS can be used as the verification platform for the relevant theory research, and provides technical reference for the project demonstration.

\section{CONCLUSION}

According to the need to the research of indirect LOS for a certain type of equipment, visual verification method of indirect LOS is studied. The method can make the relevant theoretical research results of the indirect LOS presented more intuitive, and provides a platform for algorithm. The establishment of the simulation platform greatly reduces the workload of researchers in data analysis, reduces the research threshold, and provides convenience for the research of the algorithm. The further improvement of the visual simulation technology will be helpful to the partial replacement of the actual test, and reduce the test and setting cost.

\section{REFERENCES}

[1] Ye G, Tian ZF. Research on the visualization of flight test data based on OpenGL [J]. Journal of Aeronautical Science, 2011, 32 (6): 10501057.

[2] Yin G S, Chen H Y, Zhang J, Chen J. Visual simulation of CIWS [J]. Journal of Harbin Engineering University, 2010, 31(2): 220-225.

[3] Tang Y Z, Liu H. The program design of OpenGL simulation interface for 3D missile model [J].Journal of missile and guidance, 2004,24(2):111-113

[4] Gao W W, Wang G L, Gao F Q, Gao S, Jia B. Research on the visual simulation platform of stabilized aiming system [J].Chinese Journal of testing technology, 2014,40(6):137-140.

[5] Liu H, Ma D L. UAV 3D trajectory planning and visualization simulation [J]. Journal of fire control and command, 2011, 36(7): 7274.

[6] Wang H B, Zhuang Z H, Li Y S, Gong L X. Simulation of terminal sensitive projectile fuze warhead system [J].Acta Armamentarii, 2011, 32(5):532-536.

[7] Liao S S, Wu C. Warhead power field simulation based on OpenGL [J]. Journal of Beijing Institute of Technology, 2011,31(8):888-891.

[8] Kang L, Dong C. Visual simulation research of river basin based on Performer [J]. OpenGL Journal of Huazhong University of Science and Technology (NATURAL SCIENCE EDITION), 2003,31(6):7173.

[9] Qi H, Chen H J, Wang X P, Lei M. Visual simulation of super pressure control of airship launch process [J]. Journal of Huazhong University of Science and Technology (NATURAL SCIENCE EDITION), 2007,35(9):91-93.

[10] [10] Liu L N, Wang P, Qin D G. The visual simulation of typical elements in the near earth space scene [J]. Computer engineering, 2010, 36(7):285-287 